\title{
Effect of Dynamic Center Region on the Flow and Mixing Efficiency in a New Tri-Screw Extruder Using 3D Finite Element Modeling
}

\author{
X. Z. Zhu, Y. D. He, and G. Wang \\ School of Mechanical Engineering, Liaoning Shihua University, Liaoning, Fushun 113001, China \\ Correspondence should be addressed to Y. D. He; yanddonghe@163.com
}

Received 30 April 2013; Accepted 3 September 2013

Academic Editor: Hyeong Joon Ahn

Copyright @ $2013 \mathrm{X}$. Z. Zhu et al. This is an open access article distributed under the Creative Commons Attribution License, which permits unrestricted use, distribution, and reproduction in any medium, provided the original work is properly cited.

\begin{abstract}
Three-dimensional finite element modeling of polymer melt flowing in a new co-rotating tri-screw extruder was established with mesh superposition technique. Based on the particle tracking technology, three typical particle trajectories in the tri-screw extruder were calculated using a 4th-order-Runge-Kutta method to study the dynamic motions of the particles. Then the flow visualizations in the local center region were carried out. Moreover, the dispersive, distributive and stretching mixing efficiencies of the tri-screw and twin-screw extruders were compared, respectively. The results show that when the particles move from one screw to another, there are great abrupt changes in the velocities and displacements, which induce the abrupt change in the stress magnitude. Most of particles, which are initially distributed in the inlet plane of the center region, fast flow out the outlet and don't pass through any screw. This special phenomenon induces a series of new characteristics in the residence time distribution (RTD), flow number, segregation scale and time averaged efficiency. In comparison with the twin-screw extruder, the tri-screw extruder has better mixing efficiency.
\end{abstract}

\section{Introduction}

Screw extruders are mostly used as pumping and mixing devices in polymer processing such as injection or blow molding. Their mixing efficiency and uniformity significantly affect the properties of the final product, energy consumption and costs. With the development of polymer industry, it is necessary to devise a new type of extrusion equipment with high output and better mixing efficiency. Based on the traditional single-and twin-screw extruders, a new type of triangle arranged tri-screw extruder (hereinafter referred to as tri-screw extruder) is put forward, which has three intermeshing regions and one dynamic center region [1]. Recently, the tri-screw extruder has been paid more and more attention due to its high mixing ability and output. However, as a new mixing setup for polymer processing, the complex modeling and computational simulation of the tri-screw extruder still remain very challenging, especially for the special dynamic center region with period changes of areas and geometric shapes. The mixing efficiency of the tri-screw extruder depends on its flow profiles, especially axial flow. The dynamic and period changes of areas and geometric shapes of the center region cause the local variations in the axial flow, which can affect the local and overall mixing efficiencies of the tri-screw extruder.

With the recent advances in computational fluid dynamics, it is possible to evaluate the flow in complex mixing geometries such as the twin screw and tri-screw extruders using finite element method (FEM). In particular, there are studies about the flowing and mixing mechanisms in the single and twin screw extruders with $2 \mathrm{D}$ and $3 \mathrm{D}$ models [29]. In some way, the tri-screw extruder is similar in simulation methods to the twin-screw extruder. However, the studies of flow and mixing in the tri-screw extruders are more complex than the twin-screw extruder due to their complex geometric modeling such as three intermeshing regions and one center region. In particular, the center regions have dynamically periodic areas with the three screw rotations, which give rise to great effects on the flow and mixing of tri-screw extruders. Hu and Chen [10] obtained the 3D isothermal 
flow rule in kneading blocks of the tri-screw extruder with FEM based on the CFD package, Polyflow. The mixing index and cumulative residence time distribution (RTD) in the tri-screw and twin-screw extruders were also investigated and compared. In addition, Jiang and Zhu [11] employed the simulation and experiments to verify the stronger conveying capacity in the tri-screw extruder. The high shear frequency of material in the tri-screw extruder was also found. In our previous researches, a quasi-three-dimensional modeling polymer melts flowing in a convey element of the tri-screw extruders were established using FEM. The flow characteristics and mean mixing evaluations in the tri-screw extruder were analyzed and compared with a twin-screw extruder [12]. Moreover, the temperature distributions, energy consumption, and capacity ratio in a tri-screw extruder at different screw geometric parameters and operational conditions were studied [13]. In particular, the 2D finite element modeling was used to study the effect of the dynamic center region on the flow and mixing mechanism of the tri-screw extruder. Based on the particle tracking technology, the nonlinear dynamics of a typical particle motions in the center region and the mixing process in the tri-screw extruder were analyzed with Fast Fourier transform (FFT) spectrums and Poincaré maps [14]. However, no information was presented on the effect of the dynamic center region on the mixing evaluations in the tri-screw extruder using three-dimensional modeling.

In this work, three dimensional flow and mixing characteristics of the conveying element in a novel intermeshing corotating tri-screw extruder were calculated using FEM with mesh superposition technique and Carreau-Yasuda viscous model. Firstly, the flow patterns in the tri-screw extruder, especially in the center region were analyzed. Based on the velocity fields, the typical three single-particle trajectories were tracked using a 4th-order-Runge-Kutta method to analyze the dynamic motions of material in the tri-screw extruder. Then, the flow visualizations in the special center region were carried out with particle trajectories technology. Moreover, the dispersive, distributive, and stretching mixing efficiencies in the tri-screw extruder were calculated and compared with those in the twin-screw extruder. It should be noted that the center region has fast axial velocities, which gives rise to a series of new phenomenon in the RTD, flow numbers and segregation scale, and time averaged efficiency distributions. The aim of this work is to further understand the mixing mechanism and the special contributions of the center region to the entire tri-screw extruder of axial flow and mixing efficiency with three-dimensional modeling.

\section{Modeling and Methods}

2.1. Geometry and Meshing. The tri-screw extruder investigated in this study has three screws, which array in a triangle and all clockwise rotation. In order to avoid screw interfere with one another, the numbers of screw flights must be odd, as shown in Figure 1(a). The geometric parameters of the tri-screw extruder are depicted in Table 1 . The 3D geometric modeling of the tri-screw extruder is established with AutoCAD software. The finite element (FE) models are established using Gambit software with the mesh superposition technique (MST) without remeshing for the periodical geometric change. The screw elements and barrel are meshed individually. The barrel is meshed with quadrilateral element and the screws are meshed with the combination of quadrilateral and triangular elements, as shown in Figures 2(a) and 2(b).

In order to catch the small velocity changes in the small clearances and near the walls, two boundary layer grids are employed in the FE model, as shown in Figure 2(d). At the same time, the refined grids are used in the special center region to catch the circumfluence phenomenon [13]. There are 41,997 elements and 11,166 nodes in the FE model of the tri-screw extruder, respectively. The simulations are carried out using a commercial CFD package, Polyflow. In this work, the comparisons of mixing efficiencies in the tri-screw and twin-screw extruder are employed to better understand the mixing mechanism in the tri-screw extruder. Therefore, the sizes of geometrical configurations and grid densities of the screws in the tri-screw are the same as the twin-screw extruder.

In order to check the convergence to obtain the validity of the grid-independent results for the tri-screw extruders, the refined mesh FE model is used to the refined mesh FE model of the tri-screw extruder were used to compare with the initial FE model. It can be found that the velocity magnitude curves of two kinds of meshes are almost overlapping. So the initial mesh is enough to insure the validity for the simulations.

2.2. Mathematical Models and Material. In this work, based on the quasi-steady assumption, the 3D flow conditions are considered. The governing equations of non-Newtonian flow in the tri-screw extruder with nonslip conditions are described as follows [15].

The form of the continuity and momentum equations can be expressed as

$$
\begin{gathered}
\nabla \cdot \mathbf{v}=0 \\
\nabla \cdot \boldsymbol{\sigma}+\rho \mathbf{f}=\rho\left(\frac{\partial v}{\partial t}+v \cdot \nabla v\right),
\end{gathered}
$$

where $\mathbf{v}$ the velocity vector, $\rho$ is the polymer density, $\mathbf{f}$ is the external body force per unit mass; $\boldsymbol{\sigma}$ is the stress tensor and can be expressed as

$$
\boldsymbol{\sigma}=-P \mathbf{I}+\mathbf{T},
$$

where $P$ is the isotropic pressure, $\mathbf{I}$ is the unit matrix, $\mathbf{T}$ is the extra stress tensor and can be defined as

$$
\mathbf{T}=2 \mu(\dot{\gamma}) \mathbf{D},
$$

where $\mu(\dot{\gamma})$ is the viscosity function of the local shear rate $(\dot{\gamma})$ and $\mathbf{D}$ is the rate-of-deformation tensor and is given as

$$
\dot{\gamma}=\sqrt{2 \operatorname{tr}\left(\mathbf{D}^{2}\right)}
$$

In this study, a kind of reinforced plastics, LLDPE/SBS blending melt, is used in the tri-screw extruder. This blending 


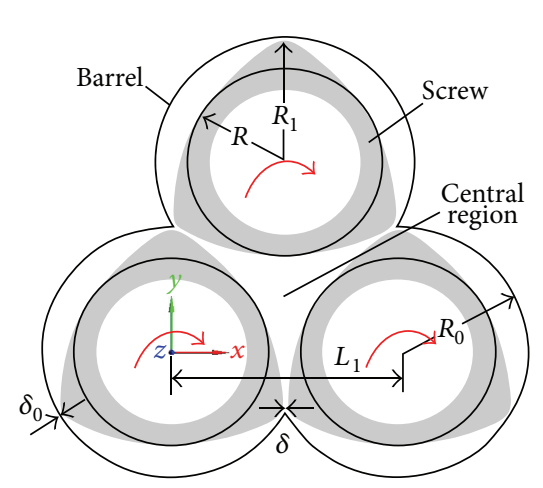

(a)

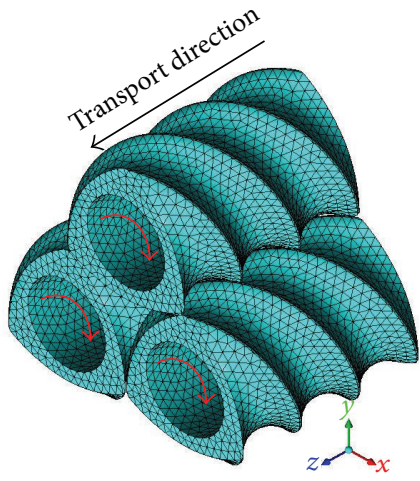

(b)

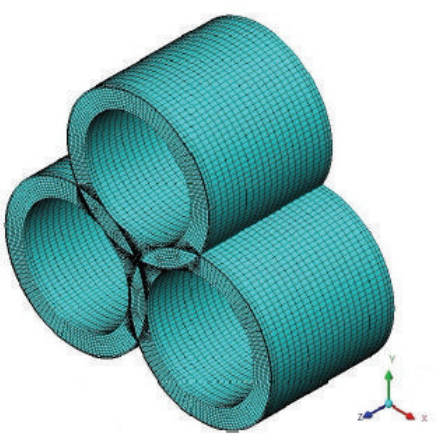

(c)

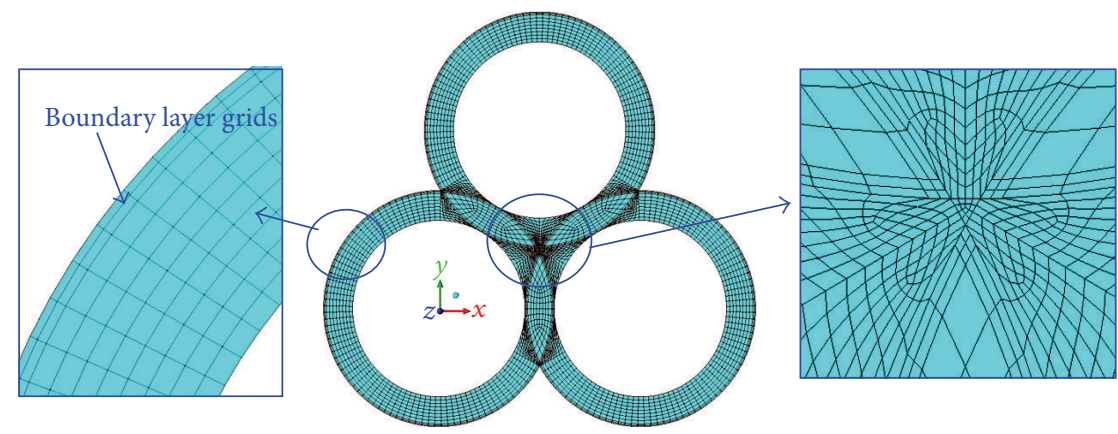

(d)

FIGURE 1: Geometric and finite element model of the tri-screw extruder: (a) geometric model in cross section; (b) grids of three screws in isometric view; (c) grids of the runner in isometric view; (d) grids of the runner in cross section.

TABLE 1: Geometrical configurations of the tri-screw extruder.

\begin{tabular}{lcc}
\hline Parameters & Symbols & Values \\
\hline Screw length & $L_{0}$ & $45.0 \mathrm{~mm}$ \\
Screw root radius & $R_{i}$ & $13.0 \mathrm{~mm}$ \\
Screw tip radius & $R_{o}$ & $17.0 \mathrm{~mm}$ \\
Barrel radius & $R_{b}$ & $17.4 \mathrm{~mm}$ \\
Centerline distance of screws & $L$ & $41 \mathrm{~mm}$ \\
Clearance of screws & $\delta$ & $0.4 \mathrm{~mm}$ \\
Clearance of screw and barrel & $\delta_{o}$ & $0.4 \mathrm{~mm}$ \\
Screw flight numbers & $n$ & 3 \\
\hline
\end{tabular}

consists of linear low density polyethylene (LLDPE) and styrene block copolymers (SBS), in which the mass ratio of the LLDPE and SBS is $50: 50$. Due to the low shear rate in the experiment, it is appropriate to use the Carreau-Yasuda model to describe the rheological behavior of the blending as follows [16]:

$$
\eta=\eta_{\infty}+\left(\eta_{0}-\eta_{\infty}\right)\left[1+(\lambda \dot{\gamma})^{a}\right]^{(n-1) / a}
$$

where $\eta_{\infty}$ is infinite shear viscosity, $\lambda$ is a model-specific relaxation time, and $\lambda=\sqrt{2 \prod_{D}} \cdot \Pi_{D}$ is the second invariant of the rate of deformation tensor and $n$ is the power-law index, which is a property of a given material. The simulation parameters of the blending melts at $160^{\circ} \mathrm{C}$ are described in Table 2.
TABLE 2: Parameters of the lending with Carreau-Yasuda model.

\begin{tabular}{lccccc}
\hline Parameters & $\eta_{0}$ & $\eta_{\infty}$ & $\lambda$ & $n$ & $a$ \\
\hline LLDPE/SBS & $5520 \mathrm{~Pa} \cdot \mathrm{s}$ & $0 \mathrm{~Pa} \cdot \mathrm{s}$ & $0.049 \mathrm{~s}$ & 0.50 & 2 \\
\hline
\end{tabular}

During the numerical calculations, the minielement and linear interpolations are used in the velocity and pressure calculations, respectively. The robust Picard iterative method is employed in the viscosity calculation due to the powerlaw index less than 0.75 . The convergence criterion is set to $1 \times 10^{-4}$ in all the simulations. The rotational speeds of three screws are $40 \mathrm{rpm}$.

2.3. Mixing Efficiency Evaluating Models. The residence time distributions are obtained with the particle tracking technique by launching virtual particles into the flow fields obtained from time dependent simulation. The motions of massless particles are dominated only by the velocity field. The trajectory path followed by a particle is obtained by the following equation:

$$
\mathbf{x}(t)=\mathbf{x}\left(t_{0}\right)+\int_{t_{0}}^{t} \mathbf{v}(\mathbf{x}) d t
$$

where $\mathbf{x}(t)$ and $\mathbf{x}\left(t_{0}\right)$ are the positions of a particle $\mathbf{X}$ at time $t$ and $t_{0}$, respectively; $\mathbf{v}(\mathbf{x})$ is the velocity vector of the particle. The fourth order Runge-Kutta method is used to track the particle trajectories in the tri-screw extruder. 

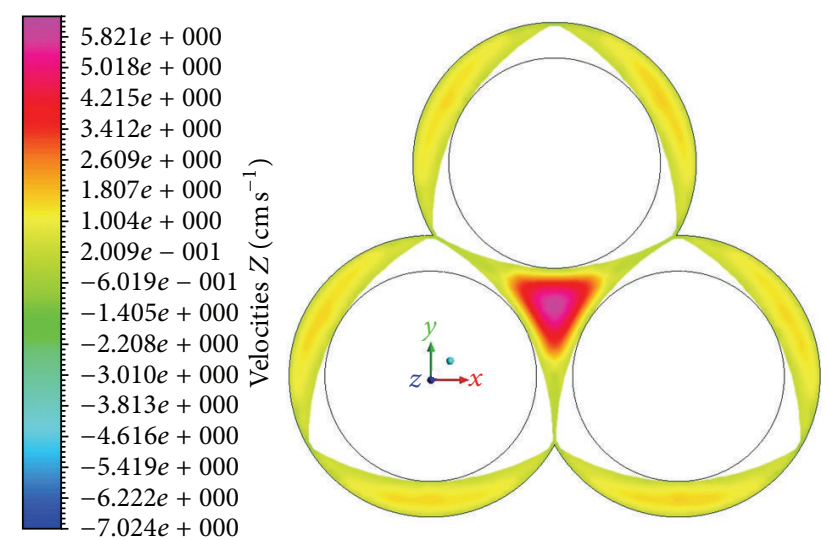

(a)
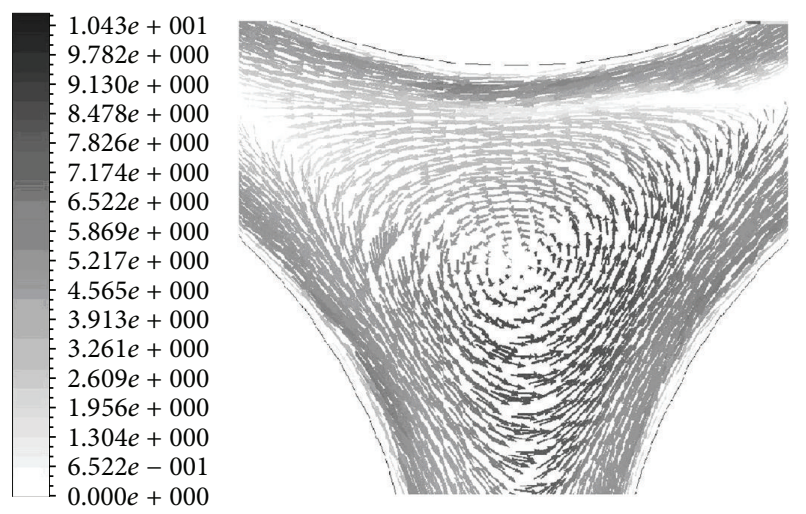

(c)

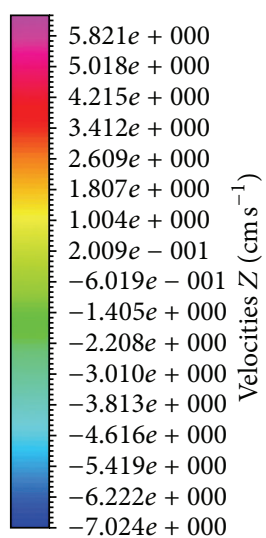

$-7.024 e+000$

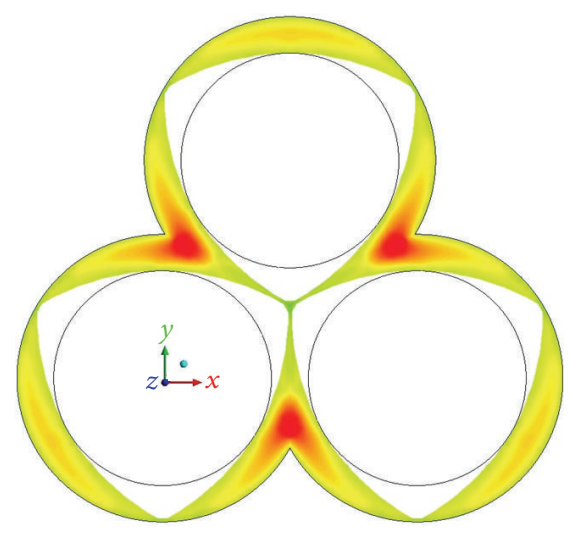

(b)
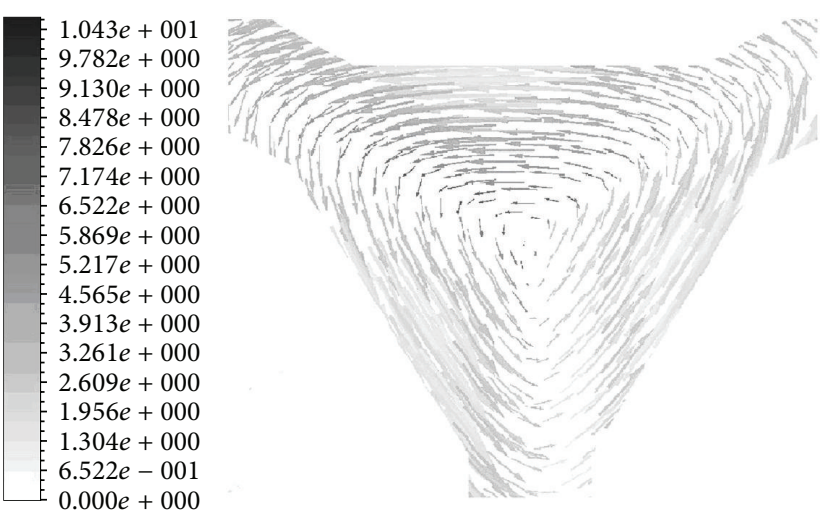

(d)

FIGURE 2: Contour map of axial velocity at different cross sections: (a) $t=0.25 \mathrm{~s}$; (b) $t=0.5 \mathrm{~s}$; (c) in center region at $t=0.25 \mathrm{~s}$; (d) in center region at $t=0.5 \mathrm{~s}$.

Residence time is an important parameter to evaluate the distributive mixing of screw extruders, which is evaluated by the residence time distribution (RTD) function, $E(t)$ and cumulative RTD function, $F(t)$. The cumulative RTD is defined as [17]

$$
F(t)=\int_{0}^{t} E(t) d t=\frac{\sum_{0}^{t} c(t) \Delta t}{\sum_{0}^{\infty} c(t) \Delta t}
$$

where $c(t)$ is the concentration of the trajectories at the exit section. $\Delta t$ is the time step. In order to accurately calculate the particle trajectories, a small iterative time step $\Delta t=0.025 \mathrm{~s}$ corresponding to $6^{\circ}$ of screw rotation is employed.

Initially, a large number of particles is distributed in some position of the flow domain. After running of the screws, the initial material particles disperse and gradually go to even distributions. The segregation scale is a measure of the size of the regions of homogenous concentration. It decreases when mixing improves and is defined as [15]

$$
S(t)=\int_{0}^{\zeta} R(r, t) d r
$$

where $R(r, t)$ is a correlation coefficient for the concentration, and it gives the probability of finding a pair of random points with a relative distance $r$ and with the same concentration.
In order to evaluate the elongation flow mixing efficiency of the inside the screw extruders, the flow number is employed and defined as [18]

$$
\lambda_{\mathrm{MZ}}=\frac{|\mathbf{D}|}{|\mathbf{D}|+|\mathbf{W}|},
$$

where $\mathbf{D}$ is the rate of deformation tensor and $\mathbf{W}$ is the norm of the vorticity tensor. The fluid in screw extruder will be in the rotational and simple shear conditions with $\lambda_{\mathrm{MZ}}=0$ and $\lambda_{\mathrm{MZ}}=0.5$, respectively, whereas the $\lambda_{\mathrm{MZ}}$ is 1.0 meaning the irrotational flow, such as pure elongational flow.

The instantaneous efficiency $e_{\lambda}$ of stretching flow is defined as a local instantaneous efficiency of mixing given by

$$
e_{\lambda}=\frac{\dot{\lambda} / \lambda}{(\mathrm{D}: \mathrm{D})^{1 / 2}},
$$

where $\mathbf{D}$ is the rate of strain tensor. The instantaneous efficiency can be thought of as the fraction of the energy dissipated locally that is used to stretch a fluid element at a given instant in a purely viscous fluid.

The time-averaged efficiency is used to describe the stretching mixing efficiency and can be defined as [19]

$$
\left\langle e_{\lambda}\right\rangle=\frac{1}{t} \int_{0}^{t} e_{\lambda} d t
$$


where $e_{\lambda}$ is the instantaneous efficiency [19]. The typical timeaveraged efficiency will decrease with the increase of the mixing time.

\section{Results and Discussion}

3.1. Flow Patterns. The tri-screw extruder in the center region has periodic geometrical configurations with screws rotation. In the present study, the mesh superposition technique (MST) is used to model tri-screw extruders without calling upon remeshing. The axial velocity distributions in the tri-screw extruder at cross-section with different time are described in Figure 2. It can be seen from Figure 2(a) that the positive axial velocity in the center region is obviously bigger than that in other regions when the area of the center region has maximal area $(t=0.25 \mathrm{~s})$. It shows a better pumping ability in the center region. Therefore, the residence time for the material experienced in the center region is shorter than that in other regions. Whereas the center region has minimal area at cross-section in Figure 2(b) $(t=0.5 \mathrm{~s})$, the values of axial velocities in the center region present negative, which reveal the backflow phenomenon in the center region. The velocity vector profiles in the center region are shown in Figures $2(\mathrm{c})$ and $2(\mathrm{~d})$. Whenever the center region has biggest or smallest area, the vertex phenomenon induced by the drag effect of three screws always exists in the center region. It should be noted that the vertex phenomenon still appears in the minimal center region, which is different from what the literature reported [11]. According to the flow number distributions, the vertex flow in the center region presents rotating flow with little flow number.

3.2. Single Particle Trajectory Behaviors. To understand the flowing and mixing mechanisms in the complex tri-screw extruder, three typical particles moving through one screw, three screws, and the center region with different residence time are selected to study their dynamic motions. Based on the particle tracking analysis, some periodical behaviors such as displacements, velocities, stretching rates, and stress magnitudes are calculated, respectively, as shown in Figures $3 \sim 5$. The dynamic analysis of them may provide useful information about the process inside the tri-screw extruder.

Figure 3 shows the periodical behaviors of Particle 1 in the tri-screw extruder, which is a typical motion moving through the three screws and only can be found in tri-screw extruder. As can be seen from Figure 4(a), the particle moving through three screws has great transverse and axial displacements with resident time of $15 \mathrm{~s}$. In Figure 3(a), each period presents this particle moving one whole turn through three screws. Each peak of the location corresponds to go through the intermeshing regions. When the particle passes through the three intermeshing regions, the motion of this particle gives rise to abrupt changes of the velocities, as shown in Figure 3(b). In comparison with velocities, the axial velocity of this particle is much less than the transverse velocity. This particle seldom presents backflow motions (negative axial velocity). on the other hand, the abrupt changes of the displacements and velocities result in the rise of stretching rates and stress magnitudes, as shown in Figures 3(c) and 3(d), respectively. The stress magnitude history in Figure 3(d) shows that this particle undergoes continuous and high stress magnitudes in the intermeshing regions, which is useful to increase the stress and stretching rates induced mixing.

Figure 4 shows the periodical behaviors of Particle 2 in the tri-screw extruder. After quickly moving $32 \mathrm{~mm}$ along the axial direction, this particle begins to move though only one screws during $5 \mathrm{~s} \sim 20 \mathrm{~s}$. The transverse displacements and velocities show regular periodical motions and no abrupt changes presents in the transverse displacement and velocity curves during moving around one screw, as shown in Figures 4(a) and 4(b). This is similar to singlescrew extruder. Correspondingly, the stretching rates and stress magnitudes undergoing by the Particle 2 are relatively small during $5 \mathrm{~s} 20 \mathrm{~s}$, as shown in Figures 4(c) and 4(d). When the displacements and velocities of this particle have abrupt changes, such as moving from one screw to another during $0 s \sim 5 \mathrm{~s}$, this particle undergoes great stretching rates and stress magnitudes. By comparison, the stress magnitude of Particle 2 moving through one screw is less than those of Particle 1 obviously, and there are only little peak values of stress magnitudes, as shown in the Figure 4(d). Therefore, the abrupt changes of displacements and velocities can give rise to the increase of stress magnitudes likely due to the chaotic motion, which is helpful to enhance the mixing efficiency.

To understand the special motions of materials in the center region, Particle 3, only moving in the center region, is selected, as shown in Figure 5. This particle in Figure 5(a) moves in the tri-screw extruder with residence time of only $2.5 \mathrm{~s}$ and little transverse displacements. However, the abrupt changes of displacements and velocities induced by the rotating screw flights result in the big values of stretching rates and stress magnitudes in the center region, as shown in Figures 5(c) and 5(d). In particular, the stress magnitudes of Particle 3 are even higher than those of Particle 1 and Particle 2, which is useful to enhance the stress and stretching induced mixing. So, the material particles in the center region have better stress induced mixing, although some vertex phenomenon existing in the center region.

3.3. Flow Visualization with Particle Trajectories. In order to investigate the convey performance of the polymer melts in the center region, 2000 particles are injected in the center region of inlet plane at $t=0 \mathrm{~s}$ ( 0 revolution), as shown in Figure 6(a). With the increase of mixing time, most of particles quickly move from inlet to outlet along the center region. When $t=1.25 \mathrm{~s}$ ( 0.83 revolutions), the first string "beads" of polymer melt arrive at the outlet plane. If the particles at inlet section are injected continuously, the consecutive strings of "beads" of polymer will come into being due to the periodic area changes of the center region. This numerical phenomenon shows a good agreement with the experiment results in Figure 6(b) by Jiang and Zhu [11]. Especially, a series of new characteristics distributed in the many mixing evaluation parameters such as dispersive and distributive mixing, are induced by this special phenomenon in the center region and will be discussed in the following 


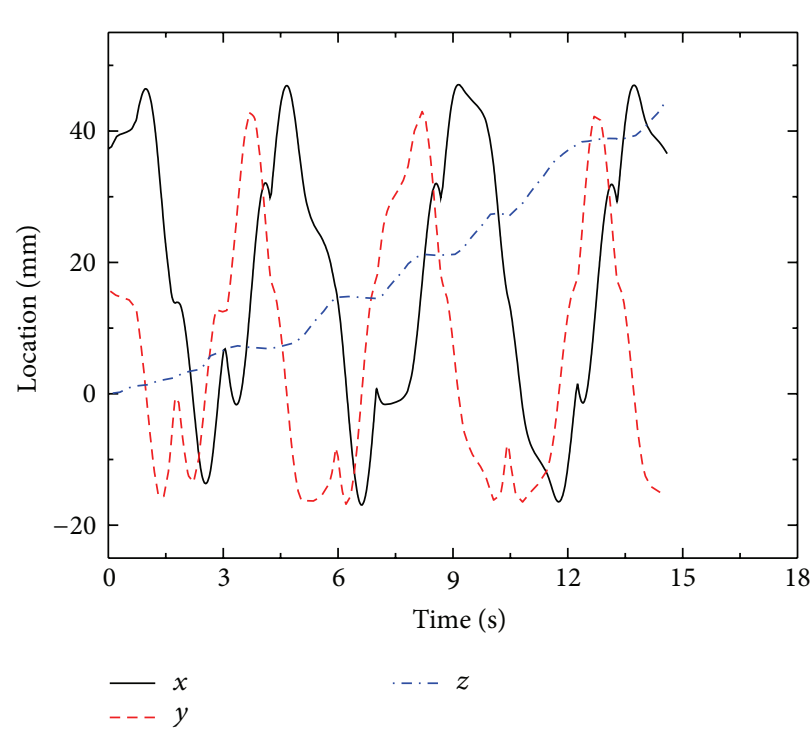

(a)

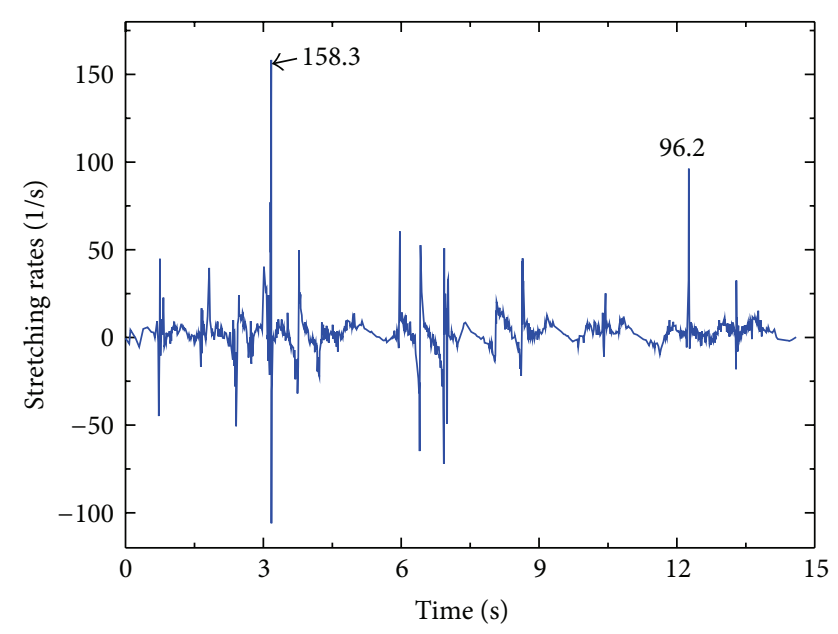

(c)

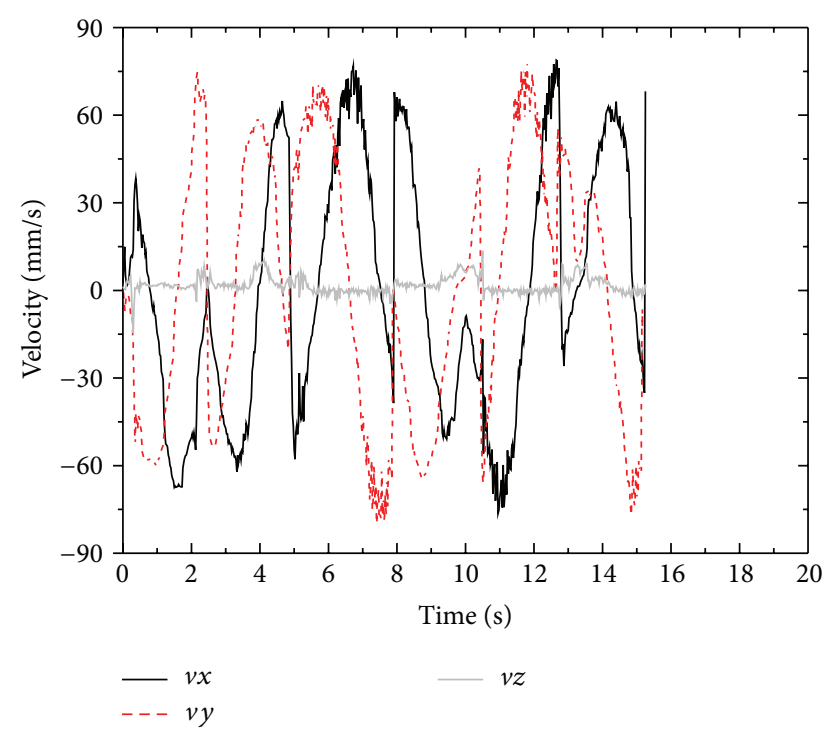

(b)

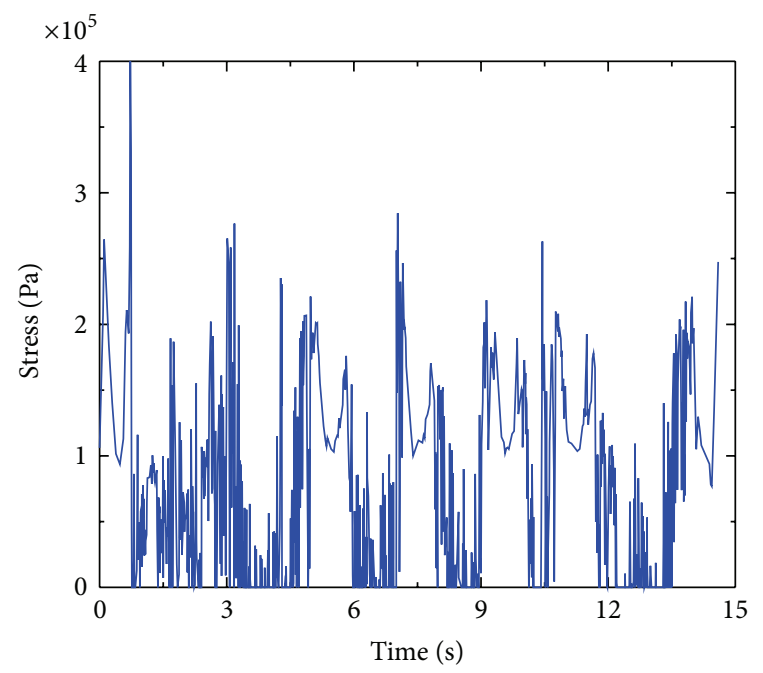

(d)

FIgure 3: Periodical behaviors of Particle 1 in the tri-screw extruder: (a) displacement; (b) velocity; (c) stretching rate; (d) stress.

sections. Please note that because the feeding position is not in the center region of tri-screw extruder, there is only little material entering into the center region and directly flow out the outlet without passing through any one screw.

3.4. Dispersive Mixing Efficiency. The elongational flow is known to be more effective than the simple shear flow for the dispersive mixing efficiency [20]. To understand the dispersive mixing mechanism in the tri-screw extruder, the comparisons of the probability of flow numbers in the twinscrew and tri-screw extruders are shown in Figure 7. When the flow numbers are between 0 and 0.6, the twin-screw extruder has bigger probability than the tri-screw extruder to obtain high flow numbers. This is because the center region has strong pumping capacity, in which the material quickly flow out the exit with little flow numbers. However, when the flow numbers are between 0.6 and 1 , the tri-screw extruder has a higher probability than the twin-screw extruder to obtain great flow numbers. In addition, the maximal flow number distributions show that the tri-screw extruder has bigger probability than the twin-screw extruder to obtain maximal flow numbers. In summary, most of polymer melts in the tri-screw extruder have great maximal flow numbers, except for the center region. This is an advantage of the triscrew extruder in the elongational mixing ability.

The stress magnitudes are important parameters to evaluate the dispersive mixing induced by stress for the triscrew extruder. Figure 8 describes the comparison of mean stress magnitude values along axial distances between the triscrew and twin-screw extruders. From Figure 8, it is clear that the polymer melts undergo higher stress magnitudes in the tri-screw extruder than in the twin-screw extruder. Therefore, the stress induced dispersive mixing efficiency is 


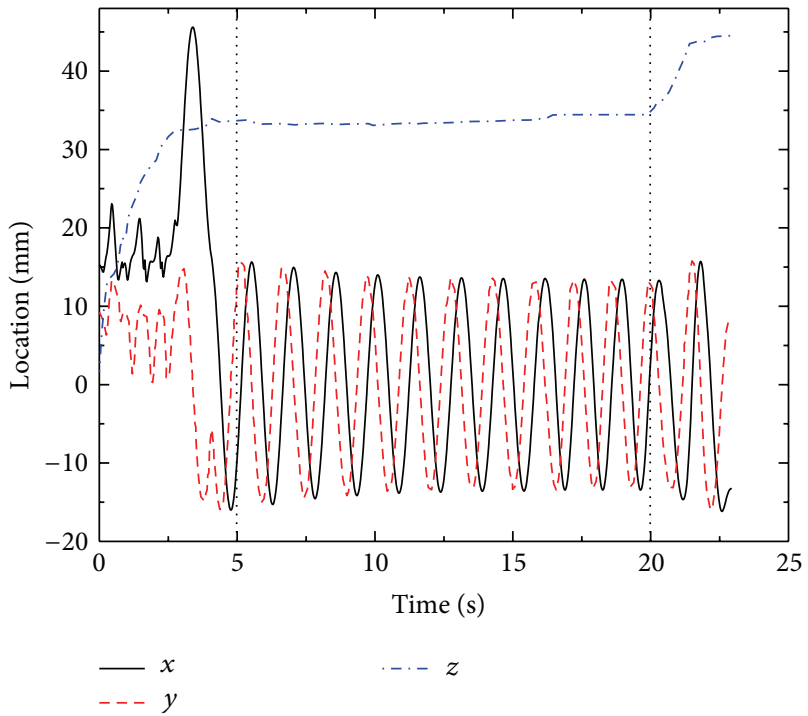

(a)

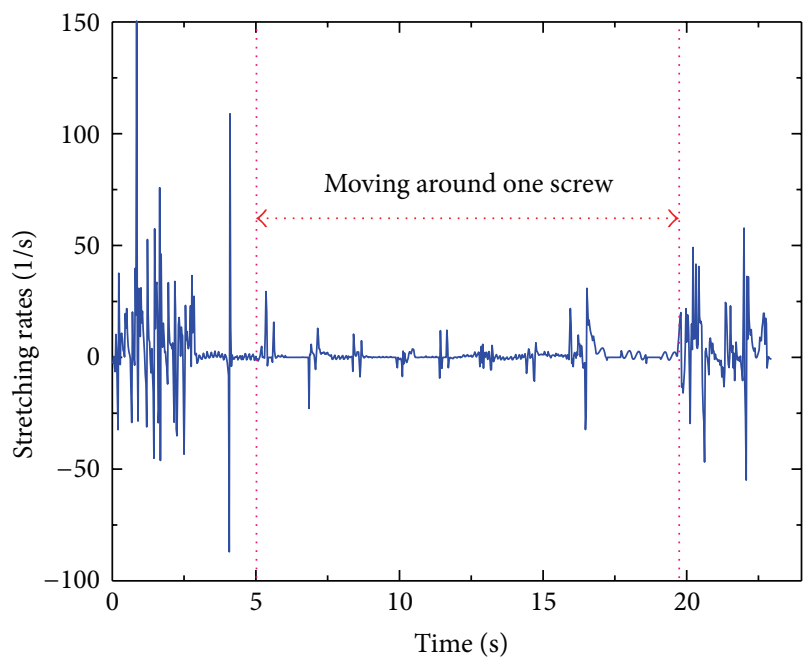

(c)

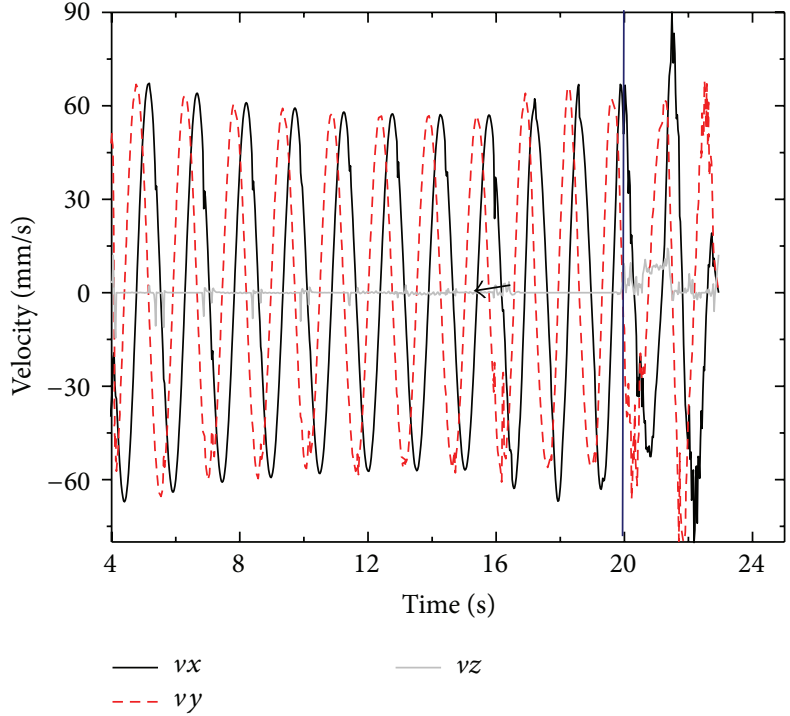

(b)

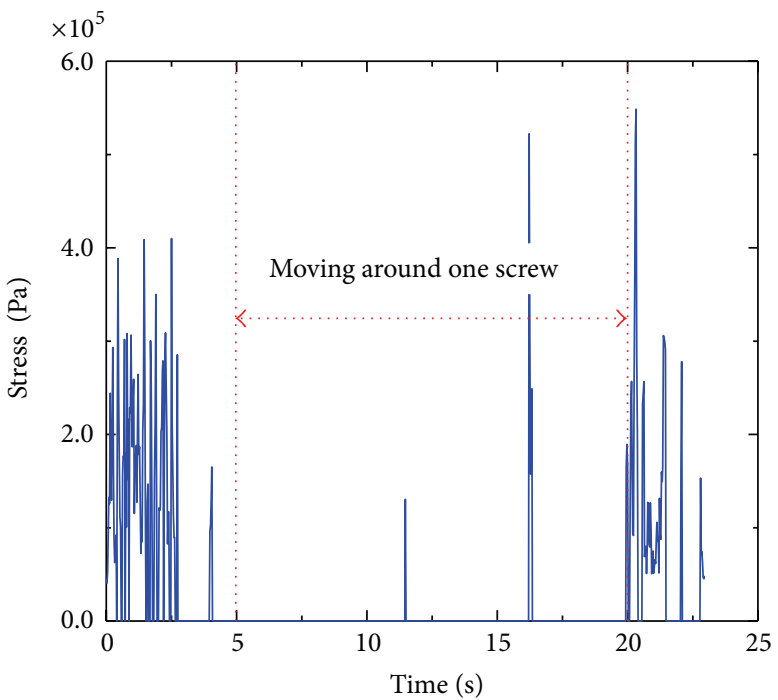

(d)

Figure 4: Periodical behaviors of Particle 2 in the tri-screw extruder: (a) displacement; (b) velocity; (c) stretching rate; (d) stress.

higher in the tri-screw extruder than the twin-screw extruder. In addition, please note that with the increase of the axial distances, the differences of the stress values between the tri-screw and twin-screw extruder increase. This is mainly because the part of polymer initial in the center region stays in the center region and has great axial velocities, which result in relative short time of experience stress effect. After $2.5 \mathrm{~s}$ (1.67 revolutions), those polymer particles (only staying in the center region) have flowed out the exit, and the rest of the particles move through the three screws or from center region into other regions with relatively long residence time and big stress magnitudes. So, the stress magnitudes in the entire triscrew extruder increase gradually along the axial distances.

3.5. Distributive Mixing Efficiency. The analysis of residence time distributions (RTD) has proved to be a useful tool to understand the distributive mixing efficiency in the mixer. The cumulative RTD and RTD are shown in Figures 9(a) and 9(b), respectively. From Figure 9(a), it can be seen that the starting point of the cumulative RTD in the tri-screw extruder is about $1.25 \mathrm{~s}$ and that in the twin-screw extruder is about $2.5 \mathrm{~s}$. At the same time, there is a platform with the slope of about zero in the cumulative RTD in Figure 9(a). It results in the two peaks in the RTD of the tri-screw extruder, as shown in Figure 9(b), which is different from the twin-screw extruder in the RTD. This is mainly because the polymer melt in the center region has bigger axial velocities than these in other regions in the tri-screw extruder. At $1.25 \mathrm{~s}(0.83$ revolutions), the first string "beads" of polymer begins to flow out the outlet. So, the starting point of the cumulative RTD in the tri-screw extruder is about $1.25 \mathrm{~s}$. During $1.25 \mathrm{~s} \sim 2.5 \mathrm{~s}$, the particles initial distributed in the center region has been 


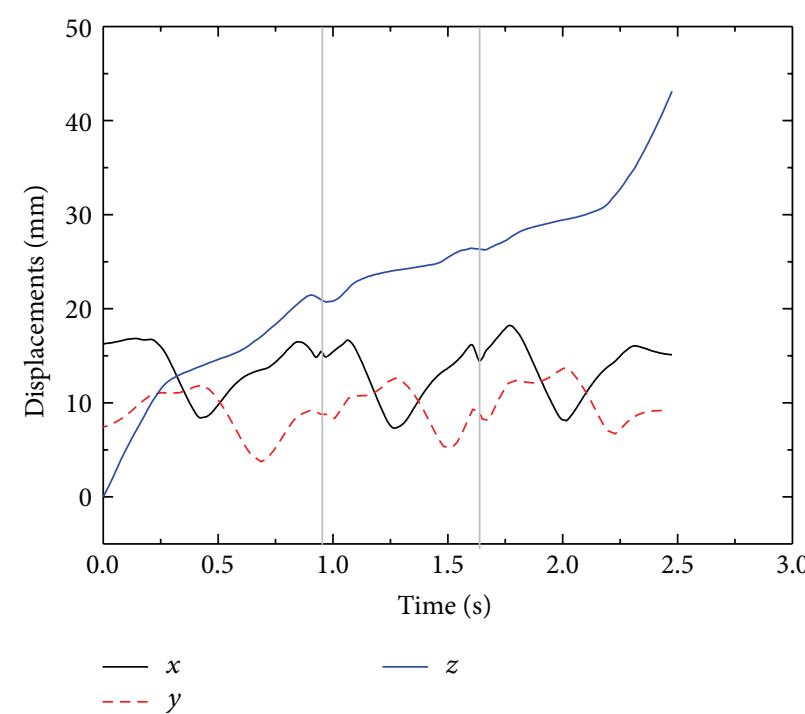

(a)

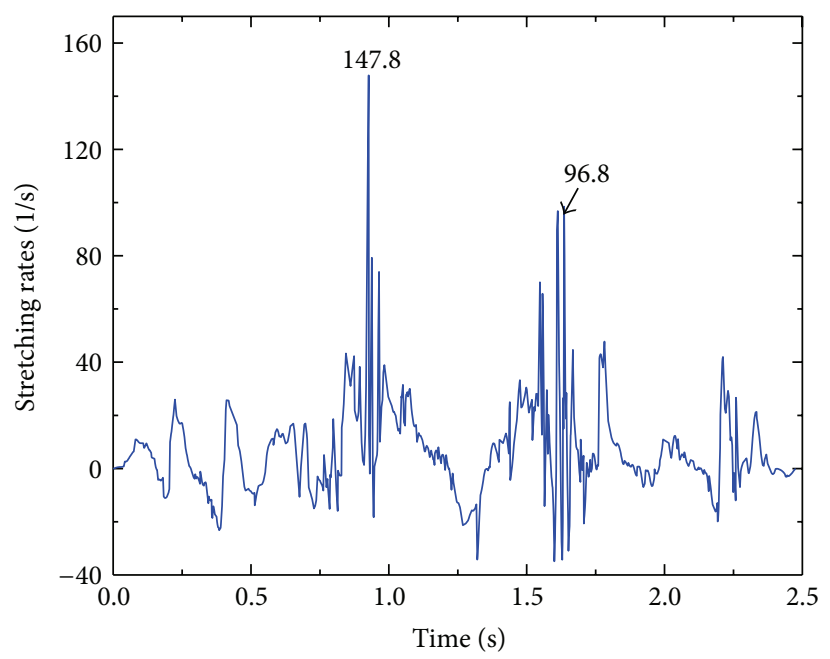

(c)

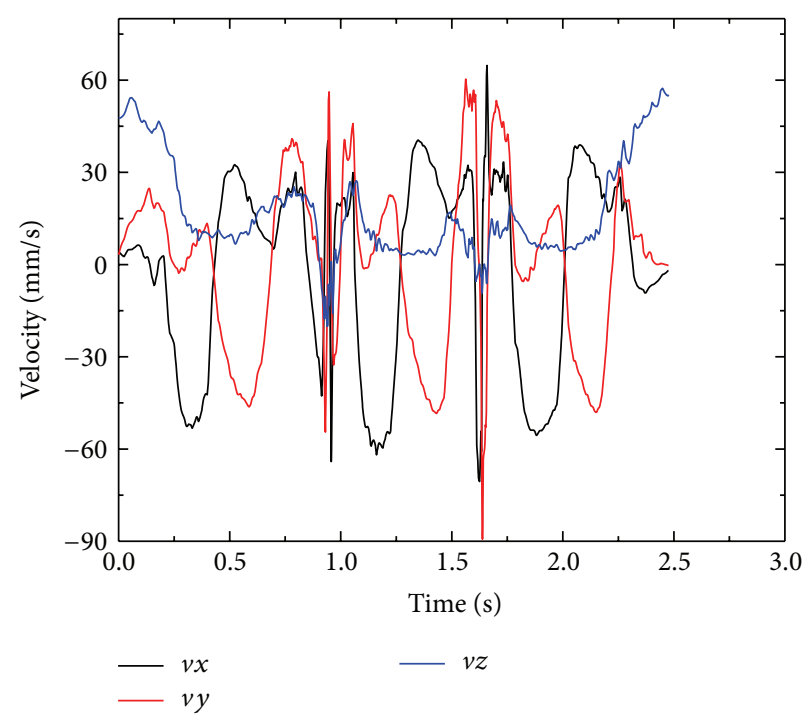

(b)

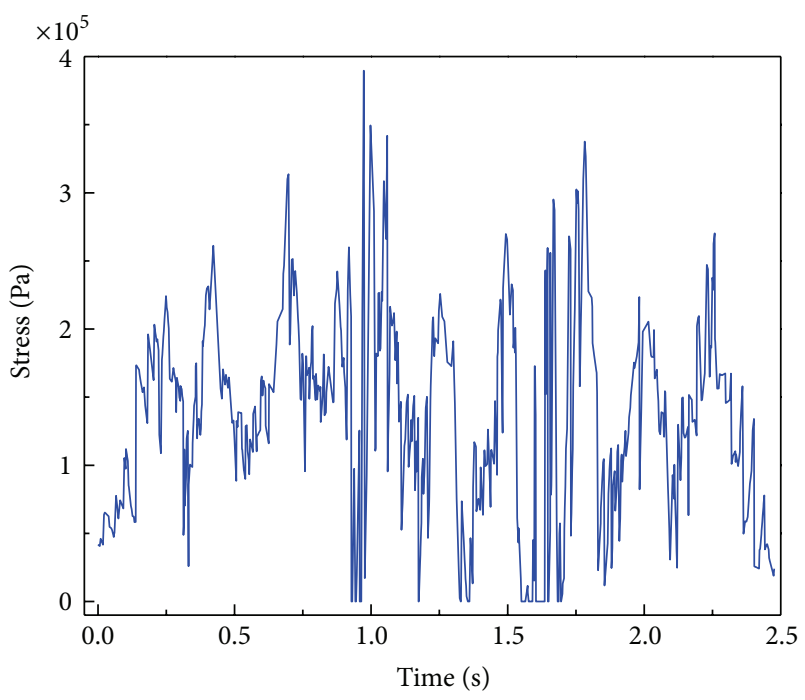

(d)

FIgure 5: Periodical behaviors of Particle 3 in the tri-screw extruder: (a) displacement; (b) velocity; (c) stretching rate; (d) stress.

moved out the exit, so the platform appears in the cumulative RTD in Figure 9(a) and the first peak of polymer appears in the RTD in Figure 9(b).

In addition, the residence time in the tri-screw extruder ranges for $1.3 \mathrm{~s} \sim 30 \mathrm{~s}$, and that in the twin-screw extruder ranges for $2.5 \mathrm{~s} \sim 24 \mathrm{~s}$. It indicates that the twin-screw extruder has better self-cleanup ability than the tri-screw extruder. on the other hand, the width of the RTD in the tri-screw extruder is larger than that of the twin screw extruder, so the tri-screw extruder has better axial distributive mixing efficiency than the twin-screw extruder.

Distributive mixing also can be evaluated by means of the segregation scale. The comparison of segregation scales between the tri-screw and twin-screw extruder are shown in Figure 10. It can be seen that the two kinds of extruders show a rapid drop during $10 \mathrm{~s}$ due to the big segregated area.
However, it is noted that there is an obvious increase of the segregation scales in the tri-screw extruder during $0.9 \mathrm{~s} \sim 1.7 \mathrm{~s}$ due to the fast axial velocities of polymer in the center region. After $10 \mathrm{~s}$, the segregation scale of the tri-screw extruder fluctuates smaller than that of twin-screw extruder. At the same time, the values of segregation scale in the tri-screw extruder are less than in the twin-screw extruder, because the center region is continually varied from big to small and disturbs the streamline. It indicates that the tri-screw extruder has better distributive mixing efficiency than the twin-screw extruder.

In order to study the local dispersive mixing efficiency in the tri-screw extruder, 2000 particles are injected in the center region (mid), at screw channel region (bellow) and near the screw edge (tip), respectively. Figure 11 shows the cluster distribution index distributions initial in above three 


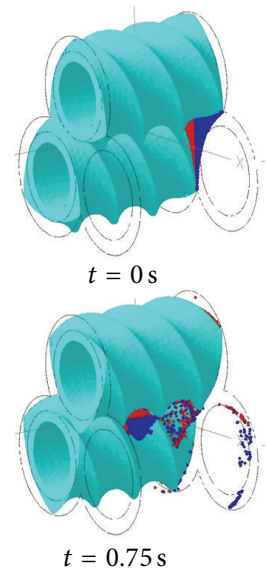

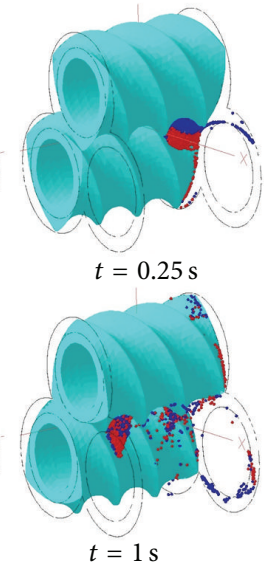

(a)

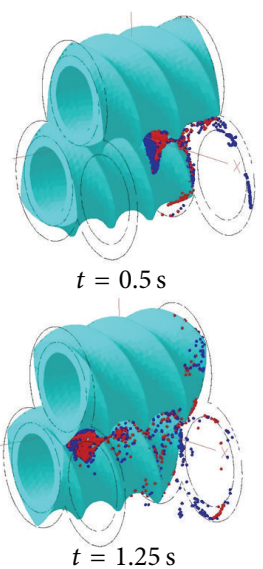

$t=1.25 \mathrm{~s}$

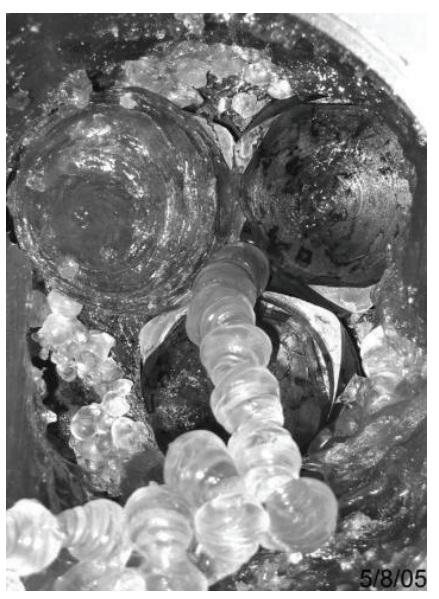

(b)

FIgURE 6: Particles conveying processing in the center region of the tri-screw extruder: (a) numerical results; (b) experiment results [11].

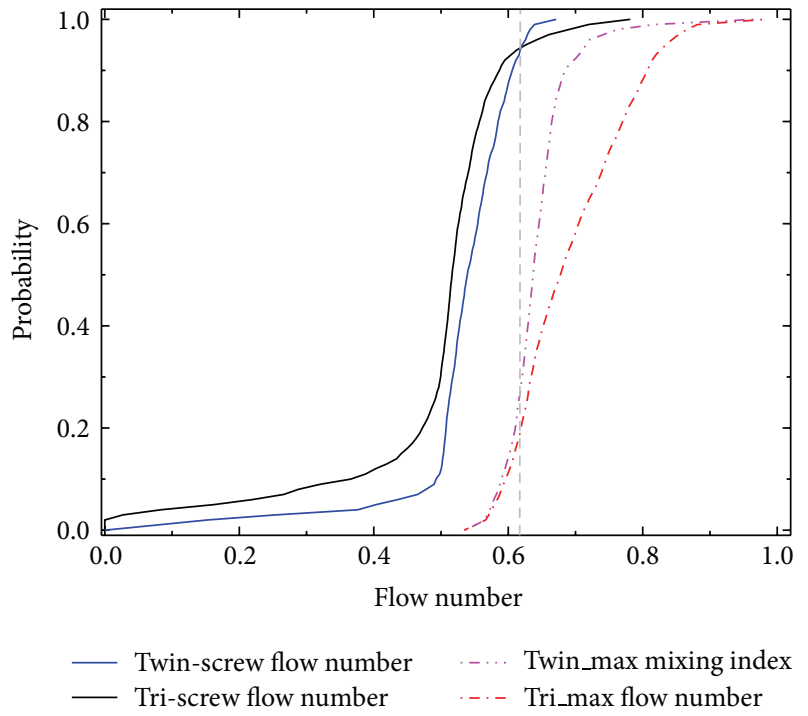

FIGURE 7: Comparisons of the probability of flow number in the two types of extruders.

regions in the tri-screw extruder. During $0 \sim 2.2 \mathrm{~s}$, the value of cluster distribution index initial in the center region is smaller than in other regions. It implies that the better mixing efficiency in the center region. This is because the particles in the center region have great axial velocities and undergo the periodic effect of shearing-squeezing-stretching by the three screws. After $5.6 \mathrm{~s}$ (3.7 revolutions), the cluster distribution index initial near the screw edge is smaller, followed by initial in the screw channel and the center region. However, it is noted that from our previous study [14], the 2D results of the cluster distribution index distributions show that the center region has minimal values of cluster distribution index than the other regions at cross-section. This difference between the $2 \mathrm{D}$ and $3 \mathrm{D}$ simulations of the cluster distribution index is because that the center region has better pumping ability, when the particles get to the outlet plane with short period of

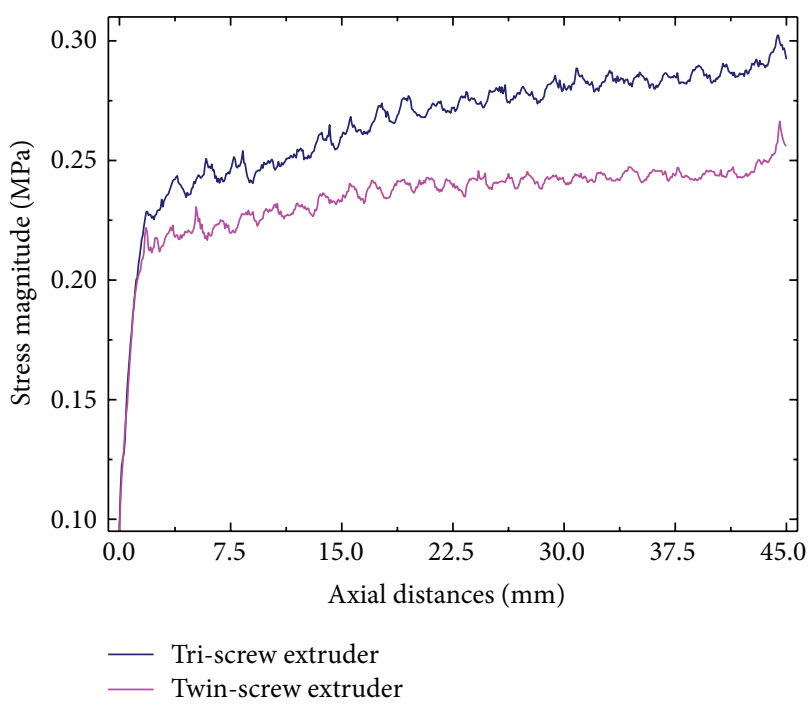

FIGURE 8: Stress magnitude distributions in two kinds of extruders.

time, their movements are equivalent to close to the wall for the cluster distribution index with 3D simulation.

3.6. Stretching Efficiency. The mean time-averaged efficiencies along the axial distances for the tri-screw and twinscrew extruder are described in Figure 12. It is shown that the quick rise in the time-averaged efficiency at the initial axial positions because the initial martial is prone to stretch due to its very small area. As the axial distances increase, the time-averaged efficiencies of the two types of screw extruders decrease and level off at a value above zero, because the stretching area of material increase. It is necessary for an effective mixer with positive value of time-averaged efficiency, indicating the strong reorientation effect. By comparison, it is found that the values of triscrew extruder are bigger than those of twin-screw extruder, which indicates that the tri-screw extruder has more efficient 


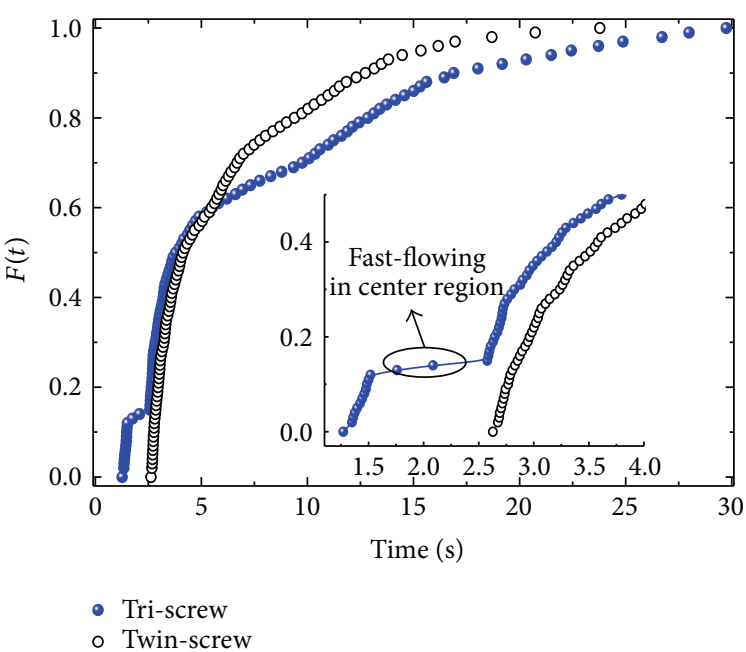

(a)

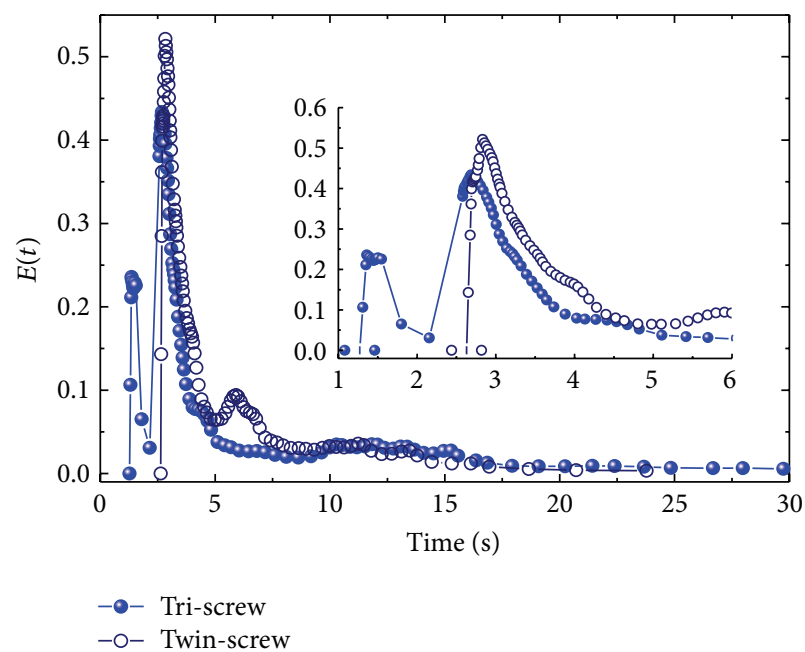

(b)

FIgURE 9: Cumulative RTD and RTD in the tri-screw and twin-screw extruders: (a) cumulative RTD; (b) RTD.

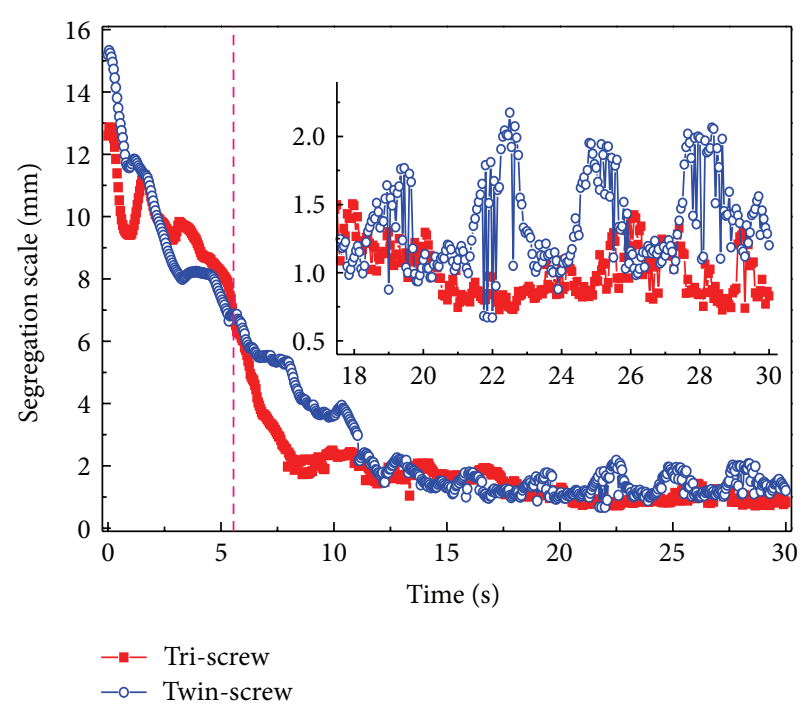

FIGURE 10: Comparison of segregation scale for the two types of extruders.

stretching mixing than the twin-screw extruder. It is noted that with increase of axial distances, the difference between the tri-screw and twin-screw extruder of the mean timeaveraged efficiency also increase because the particles initial in the center region have been moved out the outlet in the tri-screw extruder.

\section{Conclusions}

(1) The special center region of the tri-screw extruder has better pumping ability. Most of the particles initially distributed in the inlet plane of the center region quickly flow out the exit with the shape of consecutive strings "beads" and do not pass through any one screw. This special phenomenon gives rise

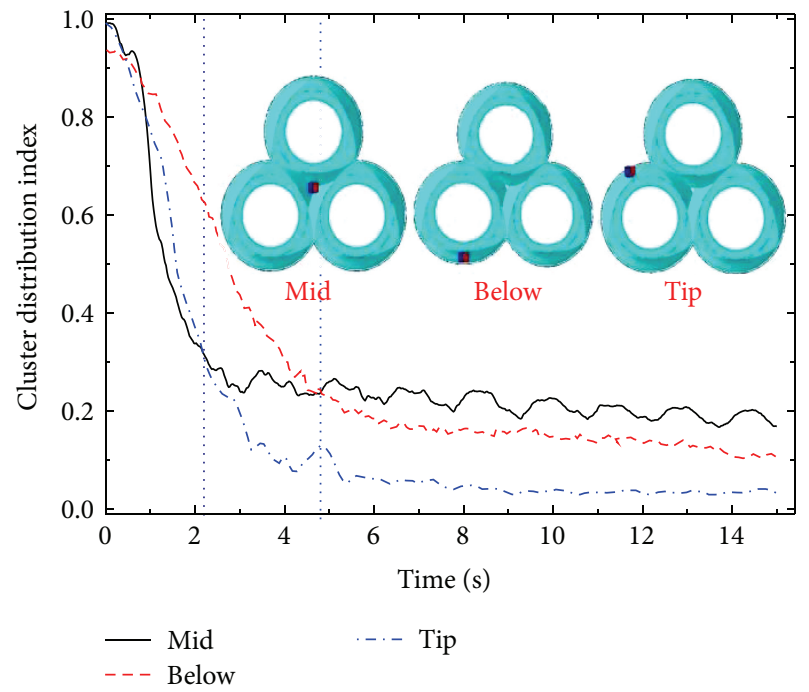

FIgURE 11: Cluster distribution index at three positions.

to a series of new characteristics in the RTD, flow numbers and segregation scale, and time averaged efficiency. When the polymer particles initially in the center region have flow out the exit, the differences of the flow numbers, segregation scale and time averaged efficiency between the twin-screw and triscrew extruders increase. In particular, there are two peaks of the RTD curve in the tri-screw extruder.

(2) When the particles move from one screw to another, there are abrupt changes of velocities and displacements, which results in the increase of the stretching rates and stress magnitudes. Because the tri-screw extruder has one more screw than the twin-screw, the particles in the tri-screw extruder have high probabilities to move from one screw to another. This helps us 


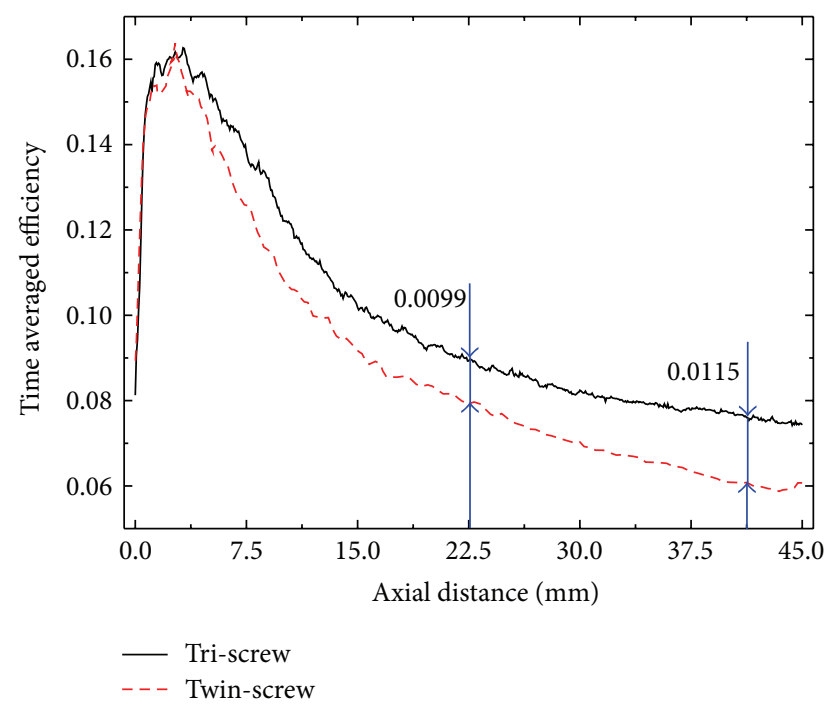

FIGURE 12: Time-averaged efficiency distributions of the two types of extruders.

to further understand the mixing mechanism in the tri-screw, twin-screw and single-screw extruders. In addition, the stress magnitudes of the particle only moving in the center region are even higher than those of the particle moving around three screws. This is beneficial to the stretching rates and stress induced mixing in the center region, although some vertex phenomenon existing in the center region.

(3) For the local mixing, the cluster distribution index in the center region is higher than that in the screw channel, implying the poor mixing efficiency in the center region due to the fast axial velocities. In practice, we can use the combined screw elements (such as conveying elements and kneading elements) to reduce the axial flow velocity and increase the material residence time in the center region to further improve the overall mixing efficiency of the tri-screw extruders.

(4) Overall, the dispersive mixing efficiency evaluations such as flow number and stress magnitudes in the entire tri-screw extruder are bigger than those in the twin-screw extruder. For the distributive mixing efficiency, the width of the RTD and in the values of segregation scale in the tri-screw extruder is larger than that in the twin screw extruder. For the stretch mixing efficiency, the tri-screw extruder has bigger mean logarithm of the area stretch than the twinscrew extruder. Therefore, the tri-screw extruder has better distributive, dispersive, and stretching mixing efficiencies than the twin-screw extruder on the overall mixing efficiency.

(5) It is noted that in this paper, the conveying element configurations of the twin-screw and tri-screw extruders were only employed. Furthermore, we will further discuss the combined screw elements of the twin screw and tri-screw extruders, such as conveying and kneading combined elements, to prove the advantage for the tri-screw extruder.

\section{Acknowledgments}

This research project was funded by the National Natural Science Foundation of China (no. 50903042 and no. 51303075), Program for Liaoning Excellent Talents in the University (no. LJQ2013041), and the Science Foundation of Liaoning Educational Committee of China (no. L2010249 and L2010247).

\section{References}

[1] N. Jiang and C. W. Zhu, "Analysis of mixing performance in a triple screw extruder," China Plastics, vol. 15, pp. 87-91, 2001.

[2] M. Kurte-Jardin, H. Potente, K. Sigge, and M. Bornemann, "Modeling the temperature development of wall-slipping polymers in single-screw channels," International Polymer Processing, vol. 24, no. 2, pp. 106-113, 2009.

[3] P. A. Moysey and M. R. Thompson, "Investigation of solids transport in a single-screw extruder using a 3-D discrete particle simulation," Polymer Engineering and Science, vol. 44, no. 12, pp. 2203-2215, 2004.

[4] R. K. Connelly and J. L. Kokini, "Examination of the mixing ability of single and twin screw mixers using 2D finite element method simulation with particle tracking," Journal of Food Engineering, vol. 79, no. 3, pp. 956-969, 2007.

[5] A. Khalifeh and J. R. Clermont, "Numerical simulations of nonisothermal three-dimensional flows in an extruder by a finitevolume method," Journal of Non-Newtonian Fluid Mechanics, vol. 126, no. 1, pp. 7-22, 2005.

[6] J. F. Hétu and F. Ilinca, "Immersed boundary finite elements for 3D flow simulations in twin-screw extruders," Computers \& Fluids, 2012.

[7] S. Ahmed Salahudeen, R. H. Elleithy, O. AlOthman, and S. M. AlZahrani, "Comparative study of internal batch mixer such as cam, banbury and roller: numerical simulation and experimental verification," Chemical Engineering Science, vol. 66, no. 12, pp. 2502-2511, 2011.

[8] H. Chen, U. Sundararaj, K. Nandakumar, and M. D. Wetzel, "On-line visualization of PS/PP melting mechanisms in a corotating twin screw extruder," International Polymer Processing, vol. 19, no. 4, pp. 342-349, 2004.

[9] M. Brito-Bazan, L. Fradette, and P. A. Tanguy, "Experimental flow visualization and residence time distributions in a cokneader," International Polymer Processing, vol. 27, no. 4, pp. 414-426, 2012.

[10] D. D. Hu and J. N. Chen, "Simulation of polymer melt flow fields in intermeshing co-rotating three-screw extruders," Journal of Beijing Institute of Technology, vol. 15, no. 3, pp. 360-365, 2006.

[11] N. Jiang and C. Zhu, "Study on extrusion characteristics of the tri-screw extruder," Polymer-Plastics Technology and Engineering, vol. 47, no. 6, pp. 590-594, 2008.

[12] X. Z. Zhu, Y. J. Xie, and H. Q. Yuan, "Numerical simulation of extrusion characteristics for co-rotating tri-screw extruder," Polymer-Plastics Technology and Engineering, vol. 46, no. 4, pp. 401-407, 2007. 
[13] X. Z. Zhu, Y. J. Xie, and Y. Miao, "Numerical study on temperature and power consumption of intermeshing corotation triangle arrayed tri-screw extruders," Polymer-Plastics Technology and Engineering, vol. 48, no. 4, pp. 367-373, 2009.

[14] G. Wang, X. Z. Zhu, Y. D. He, and L. Chen, "Effects of screw clearance and blend ratio on the flow and mixing characteristics of tri-screw extruders in the cross section with CFD," Engineering Applications of Computational Fluid Mechanics, vol. 7, no. 1, pp. 74-89, 2013.

[15] Polyflow, Release 13.0 ANSYS Inc., 2010.

[16] K. Yasuda, R. C. Armstrong, and R. E. Cohen, "Shear flow properties of concentrated solutions of linear and star branched polystyrenes," Rheologica Acta, vol. 20, no. 2, pp. 163-178, 1981.

[17] J. Gao, G. C. Walsh, D. Bigio, R. M. Briber, and M. D. Wetzel, "Residence-time distribution model for twin-screw extruders," AIChE Journal, vol. 45, no. 12, pp. 2541-2549, 1999.

[18] I. Manas-Zloczower, "Studies of mixing efficiency in batch and continuous mixers," Rubber Chemistry and Technology, vol. 67, no. 3, pp. 504-528, 1994.

[19] J. M. Ottino, W. E. Ranz, and C. W. Macosko, "A framework for the description of mechanical mixing of fluids," AIChE Journal, vol. 27, no. 4, pp. 565-577, 1981.

[20] I. Manas-Zloczower, "Analysis of mixing in polymer processing equipment," Rheology Bulletin, vol. 66, pp. 5-8, 1997. 

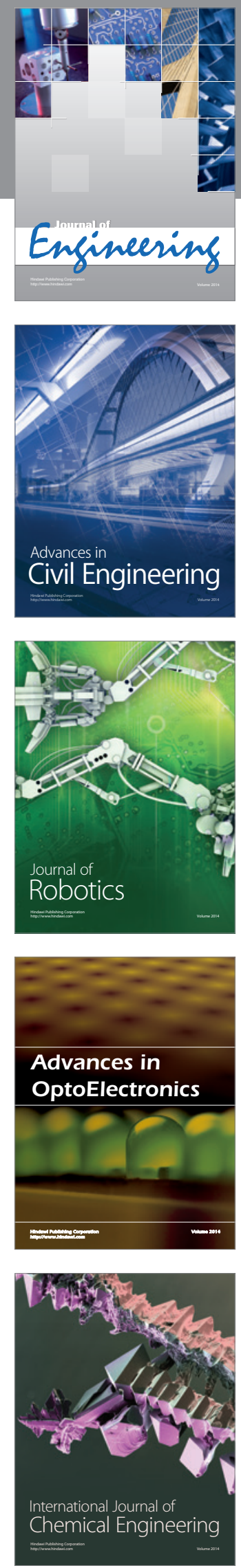

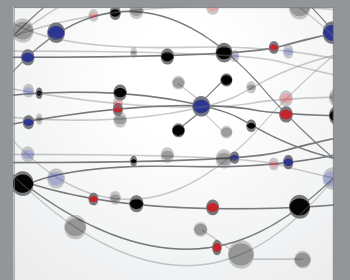

The Scientific World Journal
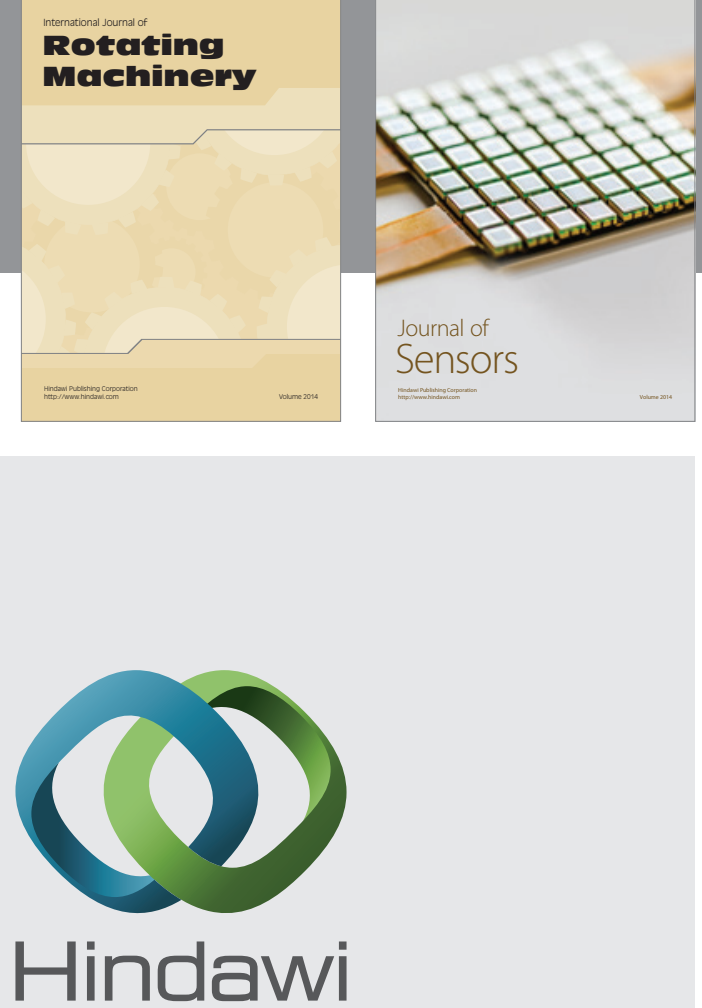

Submit your manuscripts at http://www.hindawi.com
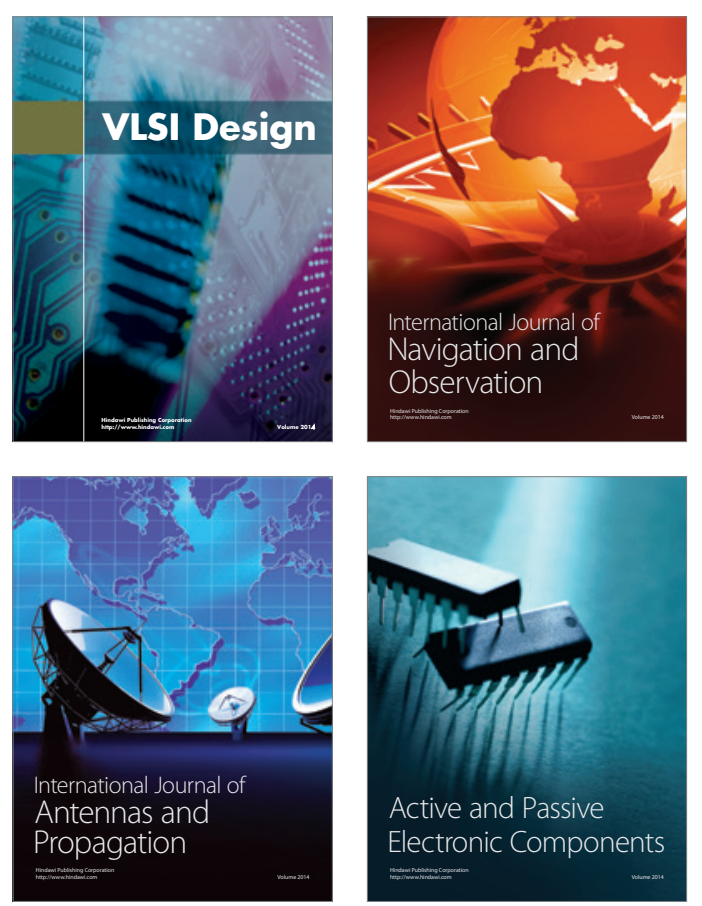
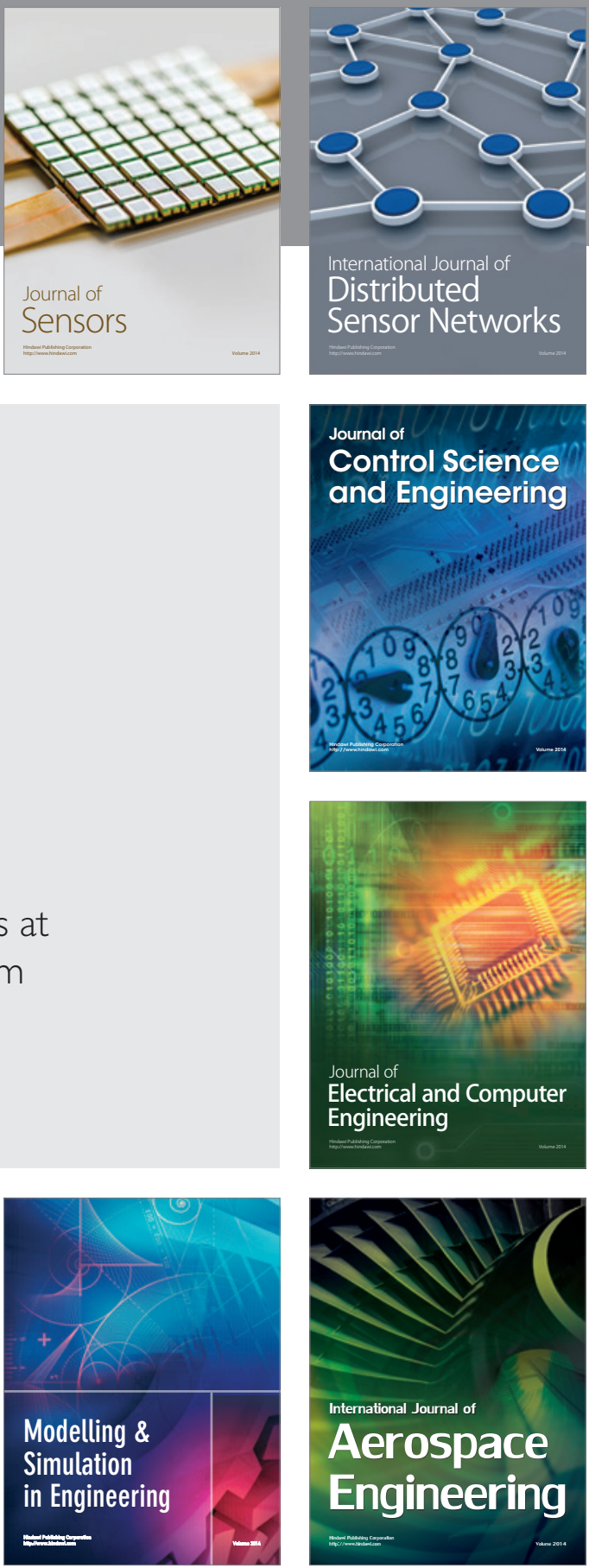

Journal of

Control Science

and Engineering
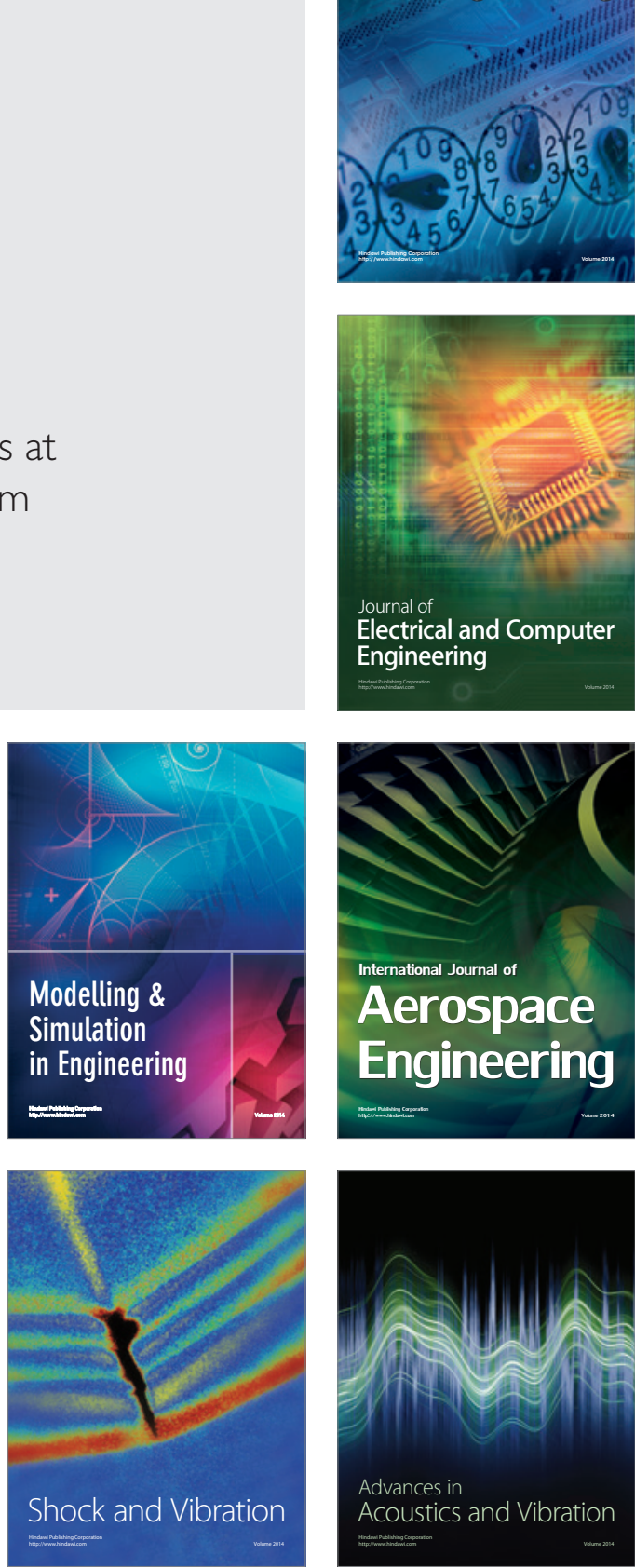